\title{
ACTIVE LASER RADAR FOR HIGH-PERFORMANCE MEASUREMENTS
}

\author{
John Hancock ${ }^{\mathrm{a}}$, Dirk Langer ${ }^{\mathrm{a}}$, Martial Hebert ${ }^{\mathrm{a}}$, \\ Ryan Sullivan', Darin Ingimarson ${ }^{\text {b }}$, Eric Hoffman ${ }^{\text {b }}$ \\ Markus Mettenleiter ${ }^{c}$, Christoph Froehlich ${ }^{c}$
}

\author{
a: The Robotics Institute, Carnegie Mellon Univ. (CMU), Pittsburgh, PA 15213, USA \\ b: $\quad$ K2T, Inc. One South Linden St., Duquesne, PA 15110, USA \\ c: $\quad$ Zoller + Froehlich $(Z+F)$, Simoniusstr. 22, D-88239 Wangen, Germany
}

\begin{abstract}
Laser scanners, or laser radars (ladar), have been used for a number of years for mobile robot navigation and inspection tasks. Although previous scanners were sufficient for low speed applications, they often did not have the range or angular resolution necessary for mapping at the long distances. Many also did not provide an ample field of view with high accuracy and high precision.

In this paper we will present the development of state-ofthe-art, high speed, high accuracy, 3D laser radar technology. This work has been a joint effort between CMU and $\mathrm{K} 2 \mathrm{~T}$ and $\mathrm{Z}+\mathrm{F}$. The scanner mechanism provides an unobstructed $360^{\circ}$ horizontal field of view, and a $70^{\circ}$ vertical field of view. Resolution of the scanner is variable with a maximum resolution of approximately 0.06 degrees per pixel in both azimuth and elevation. The laser is amplitude-modulated, continuous-wave with an ambiguity interval of $52 \mathrm{~m}$, a range resolution of $1.6 \mathrm{~mm}$, and a maximum pixel rate of $625 \mathrm{kHz}$.
\end{abstract}

This paper will focus on the design and performance of the laser radar and will discuss several potential applications for the technology. It reports on performance data of the system including noise, drift over time, precision, and accuracy with measurements. Influences of ambient light, surface material of the target and ambient temperature for range accuracy are discussed. Example data of applications will be shown and improvements will also be discussed.

Keywords: laser radar, ladar, laser scanner, imaging, inspection tasks, mobile robots

\section{INTRODUCTION}

The ability to measure surfaces and objects in 3D is becoming increasingly important for many fields such as autonomous vehicle navigation and obstacle detection, quarry mapping, landfill surveying, and hazardous environment surveying. The current state of the art for non tactile measurements is unable to meet the demand of many of these applications. Typical systems are slow and unable to measure with an unobstructed field of view.

We have created a laser radar that overcomes these limitations and provides a system that will meet the existing demand for more advanced environmental imaging. It has been developed for visual inspection tasks in both indoor and outdoor environments. The system is an optical radar, and is comparable to devices built by Erim, Odetics, and Perceptron, measuring the range between sensor and target surface as well as the reflectance of the target surface which corresponds to the power of the back scattered laser energy. In contrast to these range sensing devices, the laser radar under consideration is designed for highspeed and eyesafe measurements, emitting a minimum of near-infrared laser energy. Robustness, signal dynamic as well as accuracy and precision of range measurements of the laser radar are adapted to performance requirements of real environments.

This paper focuses on some of the design and performance issues of the laser radar in detail. In particular, the typical laser radar problem of mixed pixels is discussed as are results from accuracy and precision tests. The paper considers potential applications. Experimental results are reported.

\section{THE LASER RADAR}

The scanner device consists of a two-axis mechanical scanning mechanism in combination with a single point laser measurement system. It collects three dimensional information by steering laser energy through a 360 degree azimuth by up to 70 degree elevation field of view. 
Individual scanners can be used with different vertical field of view (the CMU scanner has a $30^{\circ}$ vertical field of view). The sensor produces a stream of $3 \mathrm{D}$ range points and intensity values arranged in a helical scan pattern. By combining a high-speed, highly accurate scanning mechanism with a high speed single point laser range sensor, we have created a data collection device which can provide remarkably high resolution range images of the sensor's physical surroundings in short periods of time.

The scanning device is comprised of a gold-coated aluminum mirror, a yoke assembly which allows the mirror to pivot vertically, a spindle assembly which rotates the yoke assembly horizontally, electronic circuits to establish the position of the mirror in space, electronic circuits to collect and store range data, a mechanical housing used to mount the range detection sensor, and the range detection device. The laser is located beneath the mirror assembly and points upwards. The scanner operates by continuously rotating the mirror in the two degrees of freedom. Data can be collected on the initial downward pivot of the mirror (downswing) and on the return sweep upwards (upswing). At each instant, the mirror deflects the laser beam at a known azimuth (determined by the rotation of the yoke assembly) and elevation (determined by the vertical position of the mirror). The laser travels outward and upon striking an object some of the scattered laser energy returns to the detector along the same path.

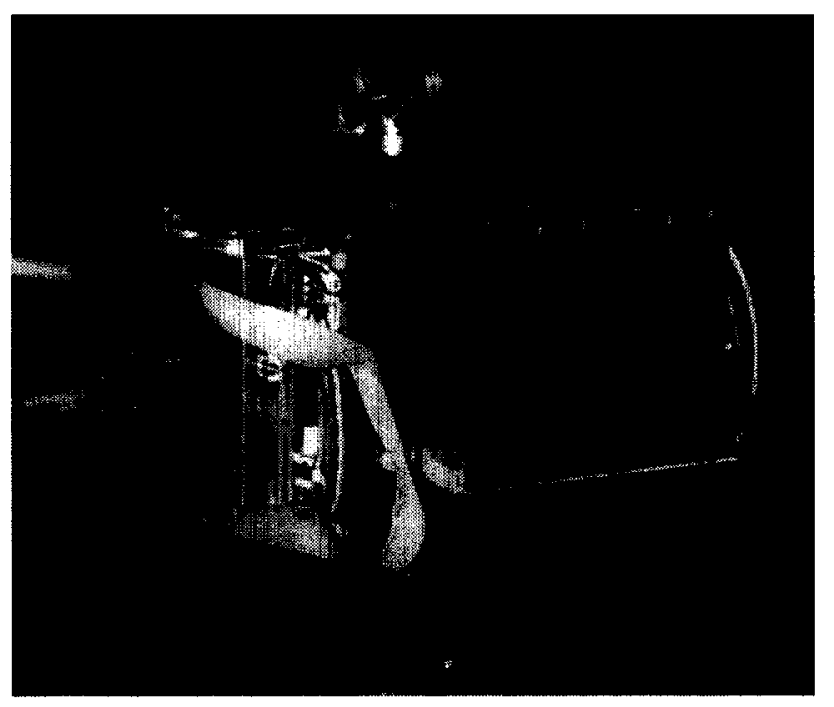

Fig. 1: High-Performance Laser Radar

The current maximum azimuth rotational speed of the scanner is $2400 \mathrm{rpm}$. Typical mirror nodding rates are 0.02 to $4.0 \mathrm{~Hz}$. The frame rate is double the mirror nodding rate since a frame can be collected on each upswing and each downswing of the mirror.
The mechanical scanner is combined with the single point $\mathrm{Z}+\mathrm{F}$ laser measurement system for 3D measurements. The laser device uses a dual frequency amplitude modulated signal and can operate at pixel rates up to (online programmable) 625,000 per second. The receiver measures the phase difference between the original and returned laser signal at both modulation frequencies and the power of the reflected laser light. The power is the reflectance whereas the phase is proportional to the range to the target, up to an ambiguity at a $360^{\circ}$ phase difference. 15-bit accuracy in the phase shift measurement of the high frequency modulation $(23 \mathrm{MHz})$ provides a range resolution of $1.6 \mathrm{~mm}$, while the phase shift in the low frequency modulation $(2.875 \mathrm{MHz})$ is used to disambiguate between multiple cycles in the high frequency, and allows for an overall ambiguity interval of $52 \mathrm{~m}$.

The laser has an overall accuracy of $+/-15 \mathrm{~mm}$ (reflectance of objects $20 \% \leq X \leq 99 \%$ ) and can see up to 100 meters (2nd ambiguity interval) in the dark and provides a 16-bit reflectance value. The scanner can provide images of up to 6000 columns by several hundred rows over the 360 by 30 degree field of view with an angular resolution of $0.06^{\circ}$ per pixel.

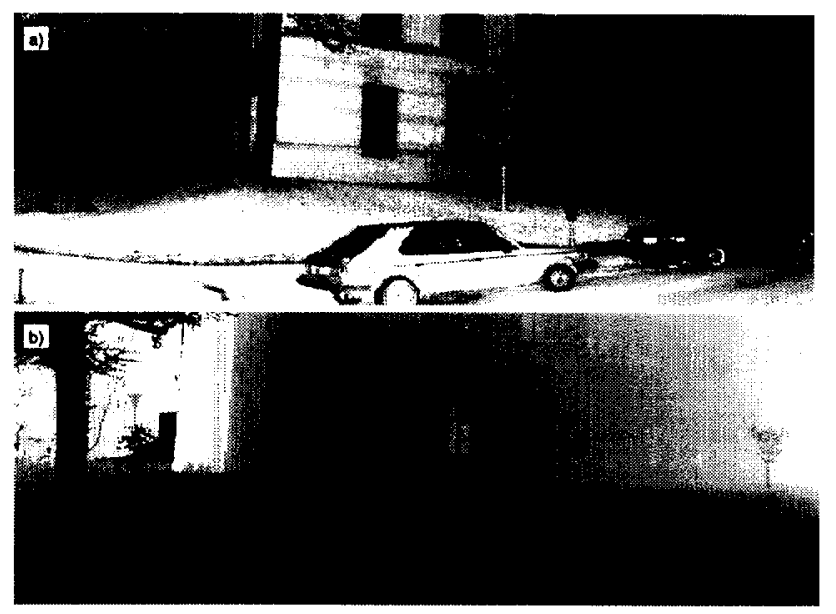

Fig. 2: Images of the laser radar (section: azimut $60^{\circ}$, elevation $30^{\circ}$ ) a) Reflectance image b) Range Image

We have subsampled the images (Fig. 2) in the horizontal direction by a factor of 3 to make them fit on to the page. In the reflectance image, top, brighter pixels correspond to points of higher reflectance. The reflectance values have been scaled for better printing. In the range image, bottom, darker pixels are closer and brighter pixels are farther. The range values have been scaled (no coordinate transformation or filtering is applied) for printing as well so that a range of 14 meters or more appears as white. 
Important Characteristics

Unlike other traditional means for obtaining depth information such as stereo vision or depth from focus, the laser scanner works in the dark as well as in ambient light. Performance is enhanced with little or no ambient light. Some versions of the scanner can be programmed on-thefly to return data from user selected regions of the scan volume. Scan speeds and sample rates can be varied to change spatial resolution and scan image sizes to better adapt to specific applications. A principal method of improving data quality is slowing the sample rate of the laser, allowing for a smaller bandwidth and effectively improving the signal to noise ratio. The highest data rates are suitable for real time monitoring of dynamic environments for security, tele-operation or navigation. Unlike camera-based systems, all data returned is fully three dimensional so structural, CAD, or solid models can be built from the raw data with post processing. The laser data is clean and dense enough to use as is for virtual walk-through once transformed to Cartesian coordinates.

This allows real-time modeling and VR applications to be satisfied with the option of storing the data for later post processing. Intensity information is also available and may potentially be used to recover some information about surface material types.

\section{Environmental Issues/Covering the Scanner}

A feature of the laser is its coaxial design. A coaxial laser has the benefit of having the transmitter and receiver fields of view overlap at all distances. This makes for more efficient laser detection at all distances. The coaxial design, however, presented a challenge when it came time to sealing the laser from the outside environment. The sensor needed a transparent cover to keep dust and dirt from collecting on optical and mechanical surfaces.

The first cover designed for the sensor was a cylindrical glass with an anti-reflective optical coating. The optical coating was designed to reduce laser reflections by $98 \%$. We discovered, however, that the glass caused problems near the center of the vertical field-of-view. Because of the coaxial design, light reflected from the glass at normal incidences was directed back at the receiver. At nearnormal incidences (-5 to 5 degrees), the laser light reflected from the inside of the glass cover overpowered the signal returned from secondary surfaces resulting in poor range values and bright reflectance values towards the center of our laser images. We surmised that although the glass resulted in laser reflection at other incidences, these reflections did not present a problem since the reflected light was not directed back at the receiver aperture.

Our second cover was designed to avoid near-normal incidence problems. We opted for an uncoated, molded optical-grade plexiglass (type P-84) design in the shape of a truncated cone. The slope of the cone was chosen to avoid normal incidences over the entire vertical field-ofview of the laser. Since the vertical field of view could be adjusted to provide laser elevation from $+5^{\circ}$ to $-25^{\circ}$, we chose the slope of the cone to be $30^{\circ}$. This shape avoided normal laser incidences over the entire field-of-view.

Unfortunately, this design also has a problem with poor data caused by laser reflections off of the plexiglass cover. In this case, the poor data occurs at the bottom of the image where there are some imperfections caused by the plastic molding process. Making a mechanical modification to the housing so that we can lower the cover further should fix the problem.

\section{PERFORMANCE RESULTS}

From a cursory visual inspection, the new scanner clearly provides better images than others. However, it is instructive to reexamine some of the problems from other laser radar scanners and see how our scanner fares. The first subsections on mixed pixels as well as range and reflectance precision and accuracy are particular to the $\mathrm{Z}+\mathrm{F}$ laser measurement system. The last subsection on angular precision is particular to the scanner mechanism itself.

\section{Mixed Pixels}

Previous AMCW laser systems have significant problems with mixed pixels. Mixed pixels are those that receive reflected energy from two surfaces separated by a large distance. Mixed pixels can result in reported ranges that are on neither surface, but somewhere between the two ranges, or even worse, either behind or in front of both surfaces. This is an inherent problem with AMCW laser radars and cannot be completely eliminated. However, the $\mathrm{Z}+\mathrm{F}$ laser reduces the problem by having a significantly smaller spot size than previous laser systems. The ERIM, for example, had a laser divergence, or instantaneous field-of-view (IFOV) of 0.5 degrees. The $Z+F$, on the other hand, has a beam divergence of only $0.5 \mathrm{mrads}$ $\left(0.03^{\circ}\right)$. Since the beam divergence is smaller than the scanner resolution, a pixel is less likely to fall on the edge of an object and thus cause an erroneous range value. When mixed pixels do occur, however, they are generally isolated pixels and may be removed by a median filter. The higher resolutions provided by the scanner make it easier to ignore or filter away isolated pixels without throwing out large amounts of the data.

\section{Range and Reflectance Precision}

To investigate the precision of the range and reflectance, data was taken with different targets (different surface material, standardized reflectances $\mathrm{X}$ ) at increasing dis- 
tances from the scanner. The scanning motors were turned off for this experiment. At each location a series of 4096 samples was taken from which the mean and standard deviation of the range and reflectance was recorded. Illumination is faint daylight (cloudy sky). Sample rate is $\mathrm{fs}_{\mathrm{s}}=500 \mathrm{kHz}$ and bandwidth is $\mathrm{fg}=140 \mathrm{kHz}$.
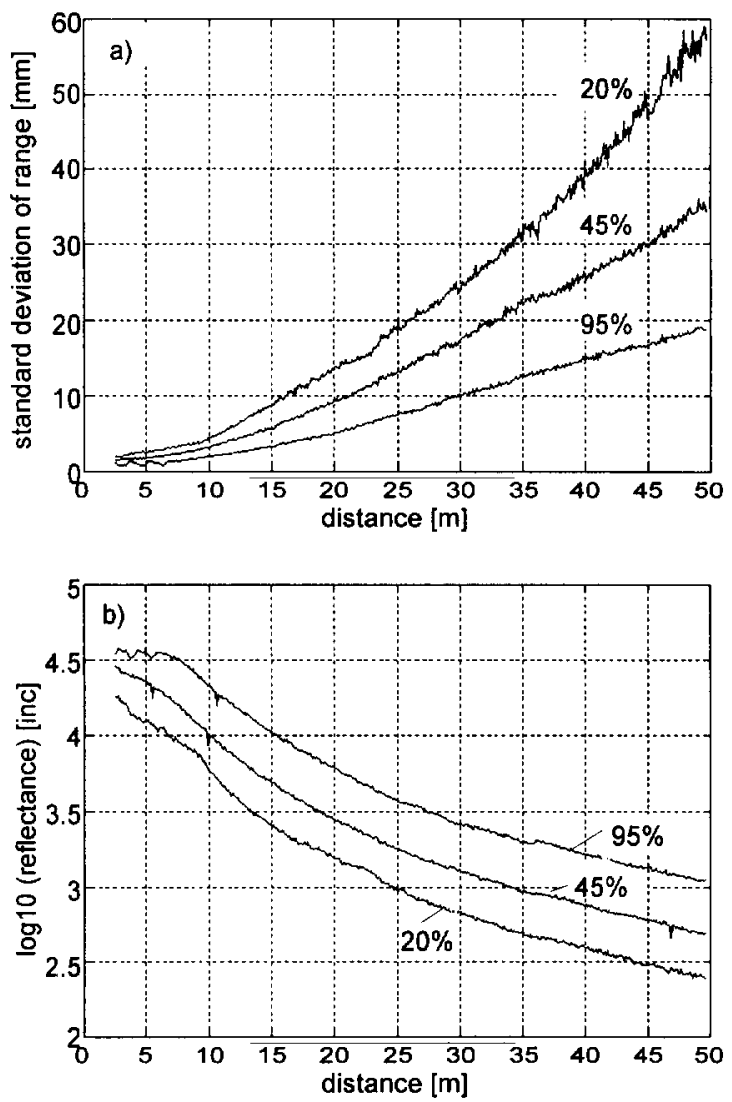

Fig. 3: Variance with reflectance

a) Standard deviation of range

b) Mean of reflectance

The system shows excellent linearity with all standardized reflectances. Figure 3a shows standard deviation of measured range samples for different surface reflectivities. It shows that standard deviation is within the whole ambuiguity interval of the measurement system less than a maximum of $60 \mathrm{~mm}(\mathrm{X}=20 \% ; \mathrm{d}=50 \mathrm{~m})$. This is excellent for high-speed laser radar measurements in mediumrange indoor and outdoor applications. It can be minimized by reducing sampling rate. Figure $3 b$ shows received magnitude of different surface reflectances over the whole ambiguity interval of the laser radar and confirms theoretical considerations. The compression of the avalanche photodiode output signal can be seen below the distance of $6 \mathrm{~m}$, especially for $\mathrm{X}=95 \%$.
Comparing figures $3 \mathrm{a}$ and $3 \mathrm{~b}$ shows that when the reflectance is high, the standard deviations of range measurements are low, but when it is weak we observe high variances. This is due to dominant noise, generated within the avalanche photodiode by ambient light.

To study the effect of ambient light on sensed range these measurements are repeated with different ambient illumination. Only when bright sunlight or a spotlight directly radiates into the laser radar, the noise of range measurement increases, therefore decreasing the precision of the measurements.
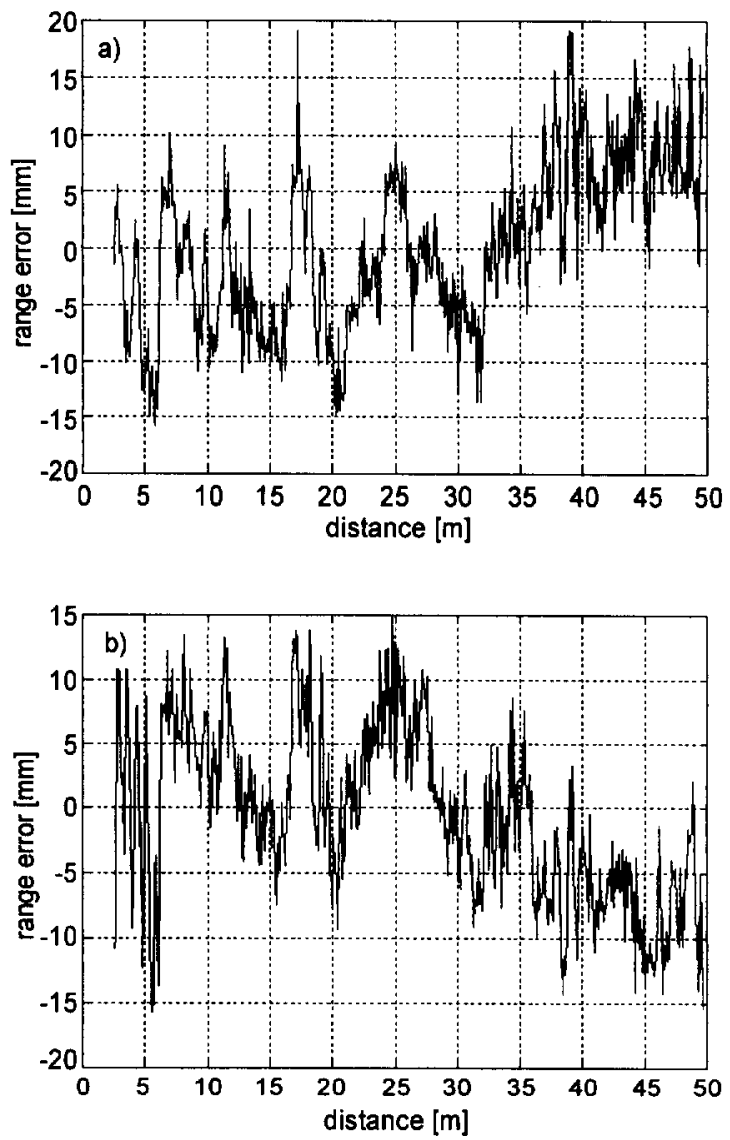

Fig. 4: Accuracy for different materials

a) Target reflectivity $X=20 \%$

b) Target reflectivity $X=95 \%$

\section{Range Accuracy}

To determine the range accuracy we acquired range measurements of targets with various surface materials and reflectance within the ambiguity interval of the laser radar. The target is positioned at known distances with an angle of incidence of about $90^{\circ}$, i.e. normal to the target surface. For each target position $(\mathrm{d}=2 \mathrm{~m}-52 \mathrm{~m} ; 10 \mathrm{~cm}$ steps) with all standardized reflectances $X$, a sample of 
4096 consecutive range measurements is acquired. Experimental results for linearity, and worst-case deviations between measured range and reference range are illustrated in Fig. 4. Without calibrating look-up-tables for both measurement channels, high accuracy with linearity (max. error $0.03 \%$ of max. range) of measurements and a worstcase range error of less than $\pm 15 \mathrm{~mm}$ for worst-case reflectance $X=20 \%$ are achieved.

To measure range drift, a single target is placed $(d=20 \mathrm{~m}$; $\mathrm{X}=50 \%$ ) in front of the laser scanner and range data is acquired over several hours. Experiments demonstrated that no significant range drift $(\Delta \mathrm{d} \leq 6 \mathrm{~mm})$ can be detected. Compared to other laser range sensors, the laser range measuring system shows good long-time stability.

To investigate the effect of ambient temperature changes, we repeat for different ambient temperatures $\left(\vartheta=0^{\circ} \ldots\right.$ $40^{\circ}$ ) accuracy measurements. Results show that temperature changes will cause an offset with range and reflectance measurements less than $5 \mathrm{~cm}$. Linearity of the system is not affected by changing temperature and therefore both offsets can be compensated easily by measuring system temperature.

\section{Angular Precision}

To measure the angular precision of the scanner, a set of 6 images was taken with a stationary scanner and scene, all on the downswing of the mirror. Six feature points were selected in the first image and matched in the remaining images. The reported azimuth and elevation angles were compared for each point and averaged over the 6 images. A more sophisticated and complete approach for measuring angular precision is described by Hebert and Krotkov. Unfortunately, we could not use this approach since the scanner provides no frame synchronization and thus image row can not be used to match features or as a measure of angular precision.

For each feature in all images, the azimuth varied by less than $+/-2$ pixels (at maximum scanner resolution) or $0.12^{\circ}$ from the average value. The elevation angle varied by less than $+1-0.6^{\circ}$ from the average. Reported elevation angles for the same feature can vary even more if the downswing elevations are compared to upswing elevation angles. This imprecision in the elevation is caused by backlash, or slop, on the vertical pivot gear for the mirror. During a single-direction scan this results in fairly small errors, but when the gear changes directions to drive the mirror in the reverse direction, the difference in swing directions results in an error of approximately $3^{\circ}$ between calculated elevation. Factory calibration could reduce this effect for the user, but has not been performed on this scanner.
For this reason, we currently only use data from the downswing of the mirror. Current versions of the scanner address this problem and improve angular resolution but have not yet been tested.

We have delayed angular accuracy tests until the elevation gear mechanism is replaced.

Experimental results of range and reflectance demonstrate high performance of the laser radar. The quantitative performance, in terms of accuracy and precision with different surface material types, fulfills the demands of surface inspection. Compared to other laser range sensors, this laser radar shows good performance data in indoor and outdoor environments, even with bright sunlight. Robustness of the laser radar is sufficient for inspection tasks in industrial environments.

\section{APPLICATIONS}

A scanner which can run at variable scan rates and provide very high resolution images (up to 6000 columns by 500 rows) in seconds could be used for a number of tasks. A few potential applications are briefly discussed below.

\section{Building Modeling}

Full interior and exterior structure and content information can be quickly generated to model or map buildings. Low resolution scans can give room shapes and obstacles in less than one second. High resolution scans can yield highly detailed models in which people, light fixtures, and even door knobs can be imaged and located (by human eyes) with ease and with no post processing. Additional processing using either custom or existing software packages can yield surface or solid models which can be rendered for visualization purposes. Floor plans can be generated as well.

\section{Surveying}

Contour maps can be generated at a rate far exceeding manual survey techniques. The scanner can be applied to quarry mapping, site mapping, stock pile estimation, cut and fill estimation, blast preparation, and a host of other standard civil engineering applications in which data must be collected.

\section{Security}

Video cameras and near IR devices can be fooled with camouflage. Even thermal imaging devices can be deceived in some cases. However, it is not possible to mask a physical volume moving through space. By scanning an area in 3D at high speed and high resolution, it is next to impossible to move across the scanned area without being detected. Fairly straightforward algorithms can be used to determine what has moved between scanned images and the relative volume of the mass which is 
moving can be calculated. This has great applications in situations where a high value asset must be guarded or a site must be monitored for intruders.

\section{Tele-operation/Dynamic Situation Monitoring}

High speed range images can be supplied in real time to an equipment operator to take the place of, or augment, 2D cameras for tele-operation applications. High resolution point clouds can show the world to the operator in 3D with fairly low computational overhead. This is most useful in environments in which video is not suitable such as night operations where lights are not possible.

\section{CONCLUSIONS AND FUTURE WORK}

In this paper we have examined in detail the measurements supplied by an amplitude-modulated laser radar. The quantitative performance, in terms of accuracy and precision with different surface material types, fulfills the demands of surface inspection. Compared to other laser range sensors, this laser radar shows good performance data in indoor and outdoor environments, even with bright sunlight. Robustness of the laser radar is sufficient for inspection tasks in industrial environments. Ambiguity interval of range measurement and range accuracy of the laser radar may be adapted to other applications by selecting other modulation frequencies.

There are a variety of areas which demand future work. First, the scanner needs to be faster to cope with the realtime requirements for autonomous vehicles. We expect that we can achieve rotational speeds of $5000 \mathrm{rpm}$. Another mechanical problem is the elevation gear backlash or slop. Gear backlash causes problems in elevation angle accuracy, especially at the transition point between the upswing and downswing of the mirror where the gear changes directions. Changing the mechanism to use a gear with a finer pitch should eliminate the problem.

Although the scanner provides visually convincing $3 \mathrm{D}$ data, we have not been able to compare the 3D data with ground truth information. More evaluation and calibration of the scanner mechanism should be performed. Although angular and range precision tests have been performed, we have not performed any angular accuracy tests. In particular, elevation accuracy needs to be tested once the gear backlash problem is solved. Although range accuracy tests with the single point device were performed, additional tests should be performed in the context of the scanner.

\section{ACKNOWLEDGEMENTS}

This research was partly sponsored by: the Ben Franklin Technology Center of Southwestern Pennsylvania, the
USDOT under Cooperative Agreement Number DTFH61-94-X-00001 as part of the National Automated Highway System Consortium, and a DARPA/TACOM grant, "Technology Enhancements for UGVs", DAAE0796-C-X075.

\section{REFERENCES}

Besl, P.: "3-D range imaging sensors". Technical Report GMR-6090, General Motors Research Lab., Warren, MI, USA, (1988)

Bishop, J. R. "Intelligent Travel: The Automated Highway System." Proceedings of the International Conference on Intelligent Autonomous Systems (IAS-3), 1993.

Eberle, K. Reflectance Processing. "Erim Staff Report." Range and Reflectance Processing Workshop Proceedings, Warren, MI, (1987).

Environmental Research Institute of Michigan, Proc. Range and Reflectance Processing Workshop, Ann Arbor, MI, (1987).

Fröhlich, C.; Mettenleiter, M.; Haertl, F.: "Imaging laser radar for high-speed monitoring of the environment" Proc. of Intelligent Transportation Systems, SPIE Symposium, Pittsburgh, (1997).

Hancock, J. High-Speed Obstacle Detection for Automated Highway Applications. Thesis proposal. Carnegie Mellon Tech. Report, CMU-RI-TR-97-17, (1997).

Hebert, M. and E. Krotkov. "3D Measurements from Imaging Laser Radars: How Good Are They?" Image and Vision Computing, vol. 10, no. 3, (1992).

Kweon, I., R. Hoffman, and E. Krotkov. Experimental Characterization of the Perceptron Laser Rangefinder. Carnegie Mellon Technical Report, CMU-RI-TR-91-1, (1991).

Nitzan, D., A. Brain, and R. Duda. "The Measurement and Use of Registered Reflectance and Range Data in Scene Analysis." IEEE Proc., Vol. 65, No. 2, (1977).

Odetics, Inc., Anaheim, California 3-D Laser Imaging System, (1989).

Perceptron, Inc., Farmington Hills, MI, LIDAR Scanning System (U.S. Patent Appl.No. 4226-00015).

VDE-Verlag, "Eyesafety for laser emitting devices". German standard, VDE-DIN EN 60825, (1993) 